\title{
openheart Serum PCSK9 is modified by interleukin-6 receptor antagonism in patients with hypercholesterolaemia following non-ST-elevation myocardial infarction
}

Thor Ueland, ${ }^{1,2,3}$ Ola Kleveland, ${ }^{4,5}$ Annika E Michelsen, ${ }^{1,2}$ Rune Wiseth, ${ }^{4,5}$ Jan Kristian Damås, ${ }^{6}$ Pål Aukrust, ${ }^{1,2,7}$ Lars Gullestad, ${ }^{2,8,9}$ Bente Halvorsen, ${ }^{1,2}$ Arne Yndestad ${ }^{1,2,9}$

To cite: Ueland T, Kleveland 0 , Michelsen AE, et al. Serum PCSK9 is modified by interleukin-6 receptor antagonism in patients with hypercholesterolaemia following non-ST-elevation myocardial infarction. Open Heart 2018;5:e000765. doi:10.1136/ openhrt-2017-000765

Received 18 December 2017 Revised 6 April 2018 Accepted 15 May 2018
Check for updates

(C) Author(s) (or their employer(s)) 2018. Re-use permitted under CC BY-NC. No commercial re-use. See rights and permissions. Published by BMJ.

For numbered affiliations see end of article.

Correspondence to DrThor Ueland; thor.ueland@ medisin.uio.no

\section{ABSTRACT}

Objective It is unclear if activation of inflammatory pathways regulates proprotein convertase subtilisin-kexin type 9 (PCSK9) levels.

Approach We evaluated (1) the temporal course of serum PCSK9 during hospitalisation following acute coronary syndrome and associations with markers of inflammation (leucocyte counts, interleukin (IL)-6, C-reactive protein) and lipid levels and (2) the effect of inhibition of IL-6 signalling with the IL-6 receptor antibody tocilizumab on PCSK9 levels in a randomised, double-blind, placebocontrolled trial release in patients with non-ST-elevation myocardial infarction.

Results Serum PCSK9 increased during the acute phase and this response was modestly associated with neutrophil counts $(r=0.24, p=0.009)$ and presence of hypercholesterolaemia $(r=0.019, p=0.045)$, but was not modified by tocilizumab. However, a modifying effect of tocilizumab on PCSK9 levels was observed in patients with hypercholesterolaemia $(p=0.024$, repeated measures analysis of variance) and this effect was strongly correlated with the decrease in neutrophils $(r=0.66$, $\mathrm{p}=0.004$ ).

Conclusions Our study suggests that patients with a more atherogenic profile may benefit from anti-IL-6 therapy with regard to PCSK9.

Trial registration number NCT01491074.

\section{INTRODUCTION}

The enzyme proprotein convertase subtilisin-kexin type 9 (PCSK9) binds to the hepatic low-density lipoprotein (LDL) receptor, thereby inhibiting recycling of this receptor resulting in attenuated removal of LDL cholesterol (LDL-C) from the circulation. ${ }^{1}$ The importance of PCSK9 for LDL-C homeostasis is illustrated in individuals with loss-of-function or gain-of-function mutations in this enzyme leading to hypocholesterolaemia or hypercholesterolaemia,

\section{Key questions}

What is already known about this subject?

- The association between proprotein convertase subtilisin-kexin type 9 (PCSK9) and inflammation is unclear.

What does this study add?

- Serum PCSK9 was not modified by anti-inflammatory treatment and not associated with change in inflammatory markers suggesting a limited influence of inflammation on PCSK9.

- In patients with an established diagnosis of hypercholesterolaemia, tocilizumab blunted the non-ST-elevation myocardial infarction-associated PCSK9 increase and this was strongly correlated with a decrease in neutrophil numbers.

How might this impact on clinical practice?

- Patients with a more atherogenic profile may benefit from anti-interleukin-6 therapy with regard to PCSK9 levels.

respectively, with dramatic effects on the incidence of atherosclerotic disease. ${ }^{2}$ Also, recent studies have shown that anti-PCSK9 therapies markedly reduce LDL-C levels leading to lower incidence of adverse cardiovascular disease outcomes in high-risk patients with hyperlipidaemia. ${ }^{134}$

PCSK9 is secreted by the liver and circulating levels could therefore be readily assessable parameter of PCSK9 status. ${ }^{5}$ In the general population, we recently found a $47 \%$ higher risk for myocardial infarction (MI) in individuals with high (ie, fourth quartile) serum PCSK9. ${ }^{6}$ There are limited data on PCSK9 in patients with acute coronary syndrome (ACS), apart from a small study with few, poorly matched, controls showing elevated levels. ${ }^{7}$ In a large study of ACS, high 
PCSK9 levels were associated with presence of hypercholesterolaemia, lipid-lowering therapy and higher C-reactive protein (CRP) levels in the acute phase, but not with 1-year mortality. ${ }^{8}$ Other studies have found higher PCSK9 levels to be associated with ischaemic events ${ }^{9}$ and severity of coronary lesions, ${ }^{10}$ suggesting that targeting of PCSK9 could have a place in the management of high LDL-C and ACS. Several studies link PCSK9 to inflammation, independent of lipids. In vitro, PCSK9 enhances inflammatory pathways in macrophages, ${ }^{11}$ augments oxidised LDL-induced apoptosis in endothelial cells ${ }^{12}$ and, in vivo, PCSK9 is expressed in human atherosclerotic plaques ${ }^{13}$ and circulating levels correlate with platelet reactivity ${ }^{9}$ and coronary plaque inflammation. ${ }^{14}$ However, whether activation of inflammatory pathways regulates PCSK9 levels is still incompletely understood.

Interleukin (IL)-6 is a pleiotropic cytokine and centrally involved in stimulating the acute phase response and orchestrating leucocyte infiltration during tissue injury. ${ }^{15}$ IL-6 has been suggested to be a major player in ACS, ${ }^{16}$ and we recently demonstrated that the humanised anti-IL-6 receptor (IL-6R) antibody, tocilizumab, had beneficial effects on inflammation (ie, CRP) and troponin $\mathrm{T}(\mathrm{TnT})$ release in patients with non-ST-elevation myocardial infarction (NSTEMI). ${ }^{17}$ To further examine the regulation of PCSK9 we analysed (1) serum PCSK9 in NSTEMI compared with healthy controls, (2) the temporal course of PCSK9 during hospitalisation relative to healthy control levels and associations with markers of inflammation, (3) effect of inhibition of IL-6 signalling with tocilizumab on PCSK9 levels in a randomised, double-blind, placebo-controlled trial, and (4) if the effect of IL-6 inhibition differs depending on cholesterol status.

\section{MATERIALS AND METHODS}

This study was part of a randomised, double-blind, placebo-controlled trial $(n=117)$ designed to evaluate the effect of a single dose of the anti-IL-6R antibody tocilizumab in NSTEMI (ClinicalTrials.gov, NCT01491074), approved by the Regional Ethical Committee, conducted according to the Helsinki Declaration with written informed consent provided by all patients. ${ }^{17}$

The study design has previously been described. ${ }^{17}$ Briefly, patients between 18 and 80 years of age with NSTEMI presumed to be caused by coronary artery disease (CAD) were eligible for inclusion. Major exclusion criteria were clinically significant heart disease other than CAD, clinical instability, inflammatory diseases or drugs affecting inflammation, contraindications to tocilizumab and any condition that could interfere with protocol adherence. Patients were included on the day of scheduled coronary angiography. After baseline blood sampling, patients received an intravenous infusion of tocilizumab $280 \mathrm{mg}$ or matching placebo (saline) prior to coronary angiography. After study drug administration and coronary angiography, six blood samples were obtained during the first 3 days of hospitalisation (day 1: evening; day 2: morning, afternoon, evening; day 3: morning, afternoon). For comparison, serum was collected from 27 apparently healthy subjects based on disease history and normal levels of CRP, recruited from the hospital staff for acquaintances.

\section{Serum PCSK9 and biochemical analysis}

Peripheral venous blood was drawn into pyrogen-free tubes without any additives. After clotting at room temperature, the tubes were centrifuged at $2100 \mathrm{~g}$ for $15 \mathrm{~min}$, and serum was stored at $-80^{\circ} \mathrm{C}$. Serum PCSK9 was measured in duplicate by enzyme immunoassay with antibodies obtained (Catalogue number DY3888) from R\&D Systems (Minneapolis, MN) in a 384-well format using a combination of a SELMA (Jena, Germany) pipetting robot and a BioTek (Winooski, VT, USA) dispenser/washer. Absorption was read at $450 \mathrm{~nm}$ with wavelength correction set to $540 \mathrm{~nm}$ using an ELISA plate reader (BioTek). The intra-assay and interassay coefficients of variations were $4.2 \%$ and $2.5 \%$ for PCSK9. Serum IL-6, high sensitivity (hs) CRP, hsTnT and lipid parameters were measured as reported previously. ${ }^{17}$

\section{Statistical analysis}

Comparison of PCSK9 levels (log transformed) between controls and patients with NSTEMI was performed using linear regression adjusting for age and body mass index (BMI). Comparison of baseline demographics and biochemical measures between the treatment groups was performed using unpaired t-tests, MannWhitney $\mathrm{U}$ tests, $\mathrm{X}^{2}$ test for independence or the Fisher's exact test depending on variable type and distribution. Univariate associations between PCSK9 and demographic, inflammatory and biochemical measures in patients with NSTEMI were assessed with Spearman's rank correlation. In cases where adjustments for study treatment and/or statin use were performed, partial correlation (using log-transformed value where appropriate) coefficients are reported. Treatment effects on PCSK9 (log transformed) were assessed using repeated measures analysis of variance (ANOVA) with baseline levels and statin use as covariates. Using this model, the group effect will indicate a treatment effect on log PCSK9 levels while the group*time interaction indicates a different temporal course. This procedure uses an unstructured covariance matrix in SPSS. To evaluate the effects of hypercholesterolaemia on treatment responses we first calculated the area under the curve (AUC) during hospitalisation (baseline to day 3) for PCSK9 using the trapezoidal rule. We then compared the treatment and hypercholesterolaemia groups (ie, four groups, placebo and tocilizumab with and without hypercholesterolaemia) with Kruskal-Wallis followed by comparisons between individual groups with Mann-Whitney U tests. Treatment effects were 


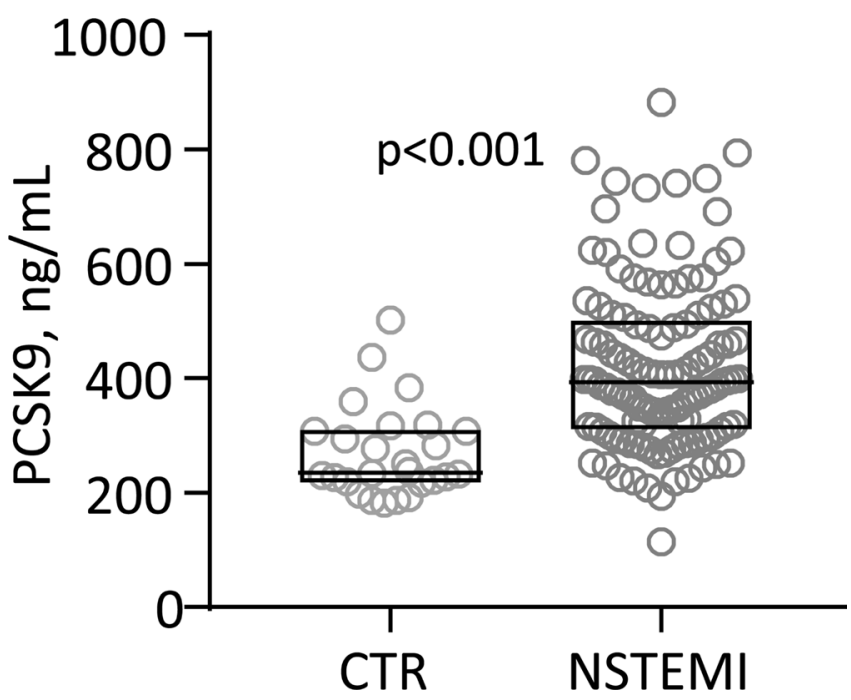

Figure 1 Tukey plot of serum proprotein convertase subtilisin-kexin type 9 (PCSK9) in healthy controls (CTR; $\mathrm{n}=27$ ) and patients with non-ST-elevation myocardial infarction (NSTEMI; $n=117$ ) on admission. $P$ values are adjusted for age and body mass index (BMI).

further analysed within the groups of patients with and without hypercholesterolaemia by repeated measures ANOVA, adjusting for baseline values and statin treatment. $\mathrm{P}$ values are two sided and considered significant when $<0.05$. Statistical analysis was performed with SPSS V.25.0.

\section{RESULTS}

\section{PCSK9 in NSTEMI}

We first compared serum PCSK9 in our patients with NSTEMI $(n=117$, mean \pm SD age $60 \pm 9$ years, $12 \%$ women, BMI $28.1 \pm 4.0 \mathrm{~kg} / \mathrm{m}^{2}, 28 \%$ smokers, creatinine $78 \pm 16$ $\mu \mathrm{mol} / \mathrm{L})$ with healthy controls $(\mathrm{n}=27$, age $66 \pm 12$ years $(\mathrm{p}=0.002), 14 \%$ women $(\mathrm{p}=0.79)$, BMI $24.9 \pm 2.7 \mathrm{~kg} /$ $\mathrm{m}^{2}(\mathrm{p}<0.001), 28 \%$ smokers $(\mathrm{p}=1.0)$, creatinine $79 \pm 12$ $\mu \mathrm{mol} / \mathrm{L}(\mathrm{p}=0.96)$ ), adjusting for differences in age and BMI, and found that patients with NSTEMI had markedly elevated levels (figure 1).

\section{Effect of tocilizumab on PCSK9}

Details regarding study population, clinical characteristics and dropouts for all patients are previously described ${ }^{17}$ and are briefly presented in table 1 showing no differences between the treatment groups.

No correlations between demographic and biochemical measures in table 1 and baseline PCSK9 were detected, except a positive correlation with BMI after adjustment for statin use. As shown in figure 2, no overall treatment effect was observed $(\mathrm{p}=0.621)$, although an interaction between treatment and time was observed $(\mathrm{p}=0.025)$. In both groups an increase in PCSK9 was observed during the acute phase with the highest levels towards the end of the second day, followed by a decline with levels similar to baseline in the placebo group. However, PCSK9 levels remained elevated compared with healthy controls throughout the observation period in both treatment groups. No significant modifying effect of percutaneous coronary intervention (PCI) was observed on the temporal course of PCSK9 (figure 2).

\section{Associations between PCSK9 and inflammatory measures during the acute phase in the total study population}

To evaluate the association between the temporal response in PCSK9 and inflammatory markers and lipids, we correlated the AUC for PCSK9 and AUC for leucocyte counts, lipid levels (total C and LDL-C), CRP, IL-6 or TnT as well hypercholesterolaemia defined as patients with an established diagnosis of hypercholesterolaemia according to prevailing Norwegian guidelines ${ }^{18}$ prior to inclusion (total $\mathrm{C}>5 \mathrm{mmol} / \mathrm{L}$ and/or LDL-C $>3 \mathrm{mmol} / \mathrm{L}$ ). A positive correlation between PCSK9 and leucocyte counts ( $\mathrm{r}=0.27$, $\mathrm{p}=0.004)$, neutrophil counts $(\mathrm{r}=0.22, \mathrm{p}=0.018)$ and presence of hypercholesterolaemia $(\mathrm{r}=0.18, \mathrm{p}=0.048)$, but not inflammatory markers or lipid levels, was detected. Leucocyte and neutrophil counts decreased during tocilizumab treatment ${ }^{17}$ and when adjusting for study treatment, the association between AUC for PCSK9 and leucocytes $(\mathrm{r}=0.20, \mathrm{p}=0.036)$ and hypercholesterolaemia $(\mathrm{r}=0.21, \mathrm{p}=0.031)$ persisted. Further adjustment for statin use revealed similar results (leucocytes, $\mathrm{r}=0.19, \mathrm{p}=0.042$; hypercholesterolaemia, $\mathrm{r}=0.21, \mathrm{p}=0.024)$. No other correlations were detected when adjusting for study treatment and/or statin use.

\section{Modifying effect of hypercholesterolaemia on the effect of tocilizumab on PCSK9}

We further explored the association between PCSK9 and hypercholesterolaemia. Comparing the AUC during hospitalisation for PCSK9 in groups stratified by treatment and presence of hypercholesterolaemia revealed a higher AUC in patients with hypercholesterolaemia treated with placebo (figure 3A). This association was further explored by comparing the temporal course of PCSK9 (figure 3B), adjusting for statin use and baseline PCSK9, and revealed no effect of tocilizumab on PCSK9 in patients without hypercholesterolaemia (top panel, $\mathrm{p}=0.97$ ). In contrast, a significant treatment effect (bottom panel, $\mathrm{p}=0.025$ ) was detected in patients with hypercholesterolaemia with a blunted increase in PCSK9 in patients treated with tocilizumab. Evaluation of associations with leucocyte counts, lipid levels, CRP, IL-6 or $\mathrm{TnT}$ within the stratification cholesterol groups revealed that the response in PCSK9 AUC strongly correlated with the AUC for leucocytes $(\mathrm{r}=0.77, \mathrm{p}<0.001)$ and neutrophils $(\mathrm{r}=0.72, \mathrm{p}=0.001)$, which both decreased during tocilizumab treatment, also after adjusting for statin use (leucocytes $r=0.64, p=0.008$; neutrophils $r=0.60$, $p=0.015$ ), in patients with hypercholesterolaemia treated with tocilizumab, but not those treated with placebo (figure 4) .

\section{DISCUSSION}

We studied the effect of the anti-IL-6R antibody tocilizumab on serum PCSK9 levels in patients with NSTEMI. 
Table 1 Baseline characteristics according to treatment group and association with PCSK9

\begin{tabular}{|c|c|c|c|c|c|}
\hline & $\begin{array}{l}\text { Placebo } \\
(n=59)\end{array}$ & $\begin{array}{l}\text { Tocilizumab } \\
(n=58)\end{array}$ & $\begin{array}{l}\text { Between groups } \\
\text { P values }\end{array}$ & $\begin{array}{l}\text { PCSK9† } \\
\mathbf{R}\end{array}$ & $\begin{array}{l}\text { PCSK9¥ } \\
\text { R }\end{array}$ \\
\hline Age (years) & $60.1 \pm 9.9$ & $59.8 \pm 7.7$ & 0.859 & -0.03 & -0.16 \\
\hline Female gender & $5(8.5 \%)$ & $9(15.5 \%)$ & 0.364 & 0.02 & 0.00 \\
\hline $\mathrm{BMI}\left(\mathrm{kg} / \mathrm{m}^{2}\right)$ & $27.4 \pm 4.4$ & $28.8 \pm 3.3$ & 0.055 & -0.08 & $0.25^{*}$ \\
\hline Hypertension & $17(28.8 \%)$ & $26(44.8 \%)$ & 0.109 & -0.09 & 0.07 \\
\hline Hypercholesterolaemia & $13(22.0 \%)$ & $17(29.3 \%)$ & 0.491 & 0.06 & 0.15 \\
\hline Diabetes type 2 & $10(16.9 \%)$ & $10(17.2 \%)$ & 1.0 & -0.06 & 0.01 \\
\hline Smoking (previous or current) & $40(67.8 \%)$ & $35(61.4 \%)$ & 0.599 & 0.06 & 0.08 \\
\hline Heart rate (beats/min) & $66 \pm 13$ & $66 \pm 10$ & 0.770 & -0.08 & 0.02 \\
\hline $\mathrm{SBP}(\mathrm{mm} \mathrm{Hg})$ & $137 \pm 18$ & $140 \pm 18$ & 0.389 & -0.06 & 0.02 \\
\hline $\mathrm{DBP}(\mathrm{mm} \mathrm{Hg})$ & $81 \pm 12$ & $83 \pm 12$ & 0.273 & -0.05 & 0.08 \\
\hline $\mathrm{PCl}$ & $47(79.7 \%)$ & $41(70.7 \%)$ & 0.367 & -0.04 & 0.02 \\
\hline CABG & $7(11.9 \%)$ & $6(10.3 \%)$ & 1.0 & 0.00 & 0.12 \\
\hline Medical treatment & $5(8.5 \%)$ & $11(19.0 \%)$ & 0.167 & 0.05 & -0.14 \\
\hline GRACE score & $92(75,105)$ & $86(72,97)$ & 0.168 & 0.00 & -0.16 \\
\hline \multicolumn{6}{|l|}{ Biochemistry } \\
\hline Creatinine $(\mu \mathrm{mol} / \mathrm{L})$ & $76(69,90)$ & $76(66,83)$ & 0.260 & -0.10 & 0.02 \\
\hline Leucocytes $\left(\times 10^{9} / L\right)$ & $7.3(5.9,9.2)$ & $7.9(5.9,9.3)$ & 0.406 & 0.12 & 0.02 \\
\hline Neutrophils (×109/L) & $4.5(3.4,6.0)$ & $5.1(3.4,6.3)$ & 0.471 & 0.08 & 0.04 \\
\hline Interleukin-6 (pg/mL) & $2.2(1.1,4.6)$ & $2.7(1.2,8.7)$ & 0.185 & 0.02 & -0.10 \\
\hline C-reactive protein (mg/L) & $2.5(1.0,8.3)$ & $2.7(1.5,4.7)$ & 0.458 & 0.02 & 0.01 \\
\hline Troponin T (ng/L) & $192(71,571)$ & $128(53,749)$ & 0.965 & 0.05 & 0.05 \\
\hline Total cholesterol (mmol/L) & $5.3(4.5,5.9)$ & $4.9(4.2,5.7)$ & 0.279 & -0.02 & 0.10 \\
\hline LDL cholesterol (mmol/L) & $3.2(2.8,3.8)$ & $3.0(2.3,3.6)$ & 0.149 & -0.09 & 0.09 \\
\hline \multicolumn{6}{|l|}{ Medication at baseline } \\
\hline Aspirin & $59(100 \%)$ & $57(98.3 \%)$ & 0.496 & 0.04 & 0.06 \\
\hline Clopidogrel & $32(54.2 \%)$ & $32(55.2 \%)$ & 1.0 & -0.02 & -0.06 \\
\hline Ticagrelor & 27 (45.8\%) & $26(44.8 \%)$ & 1.0 & 0.02 & 0.06 \\
\hline Low molecular weight heparin & $54(91.5 \%)$ & $51(89.5 \%)$ & 0.952 & 0.01 & -0.03 \\
\hline Beta blocker & $45(76.3 \%)$ & $45(77.6 \%)$ & 1.0 & -0.05 & -0.05 \\
\hline Statin & $53(89.8 \%)$ & $53(91.4 \%)$ & 1.0 & 0.14 & - \\
\hline
\end{tabular}

${ }^{*} \mathrm{P}<0.01$.

†Spearman correlation.

$\ddagger$ Partial correlation adjusting for statin use. Categorical data are given as $\mathrm{n}(\%)$. Continuous data are expressed as mean \pm SD or median (25th, 75th percentiles) and were compared with unpaired parametric or non-parametric tests depending on distribution.

$\mathrm{BMI}$, body mass index; CABG, coronary artery bypass grafting; DBP, diastolic blood pressure; GRACE, Global Registry of Acute Coronary Events; LDL, low-density lipoprotein; PCI, percutaneous coronary intervention; PCSK9, proprotein convertase subtilisin-kexin type 9; SBP, systolic blood pressure.

Patients with NSTEMI had increased serum PCSK9 compared with controls and levels increased on the first 2 days during hospitalisation with no differences between the treatment groups. However, stratifying patients revealed that tocilizumab attenuated the response in PCSK9 during hospitalisation in patients with an established diagnosis of hypercholesterolaemia prior to NSTEMI and study enrolment.

A recent study reported elevated PCSK9 in patients with ACS compared with healthy controls, although the control group was reported as 'young' and no age was given. ${ }^{7}$ Our findings demonstrate that patients with NSTEMI are characterised by increased PCSK9 levels compared with a more evenly matched healthy control group. Almontashiri et al reported that PCSK9, evaluated on admission, was elevated in acute MI compared with CAD without MI or with prior MI, suggesting a transient increase during ACS. ${ }^{19}$ However, our longitudinal assessment argues against differences between patients with ACS at admission and patients with stable CAD as we 


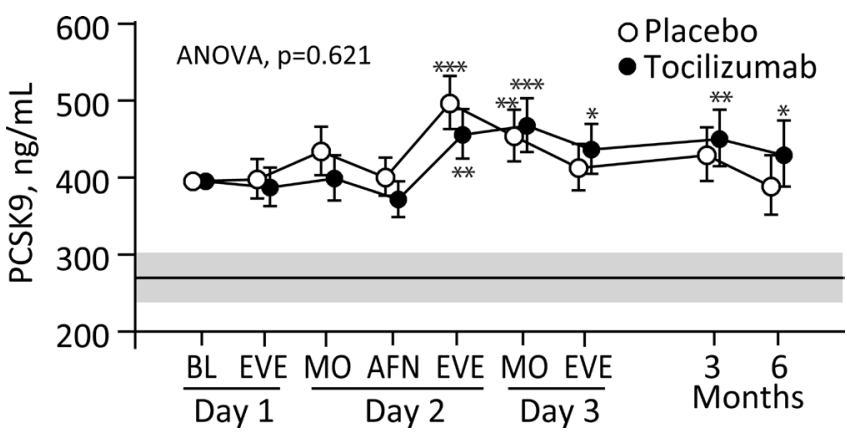

Figure 2 Serum PCSK9 in patients with non-STelevation myocardial infarction (NSTEMI) receiving placebo $(n=59)$ or tocilizumab $(n=58)$ during hospitalisation and at 3 and 6 months of follow-up. Circles represent geometric back-transformed estimated marginal means and 95\% Cls, respectively. ${ }^{*} \mathrm{P}<0.05 ;{ }^{* *} \mathrm{P}<0.01 ;{ }^{* * *} \mathrm{P}<0.001$ versus baseline $(\mathrm{BL})$. The grey area and line represent the geometric mean and $95 \% \mathrm{Cl}$ for healthy controls. AFN, afternoon; ANOVA, analysis of variance; EVE, evening; MO, morning; PCSK9, proprotein convertase subtilisin-kexin type 9 .

observed similar levels of PCSK9 at baseline and at 3 and 6 months of follow-up. Nonetheless, we also show that PCSK9 displayed a transient response during hospitalisation with a maximum increase on day 2 , with no relation to PCI, in agreement with the only previous study evaluating the temporal course in ACS. ${ }^{10}$

Clinical and experimental studies show that systemic inflammation increases the expression of PCSK $9^{20}$ and that PCSK9 increases with severe traumatic injury. ${ }^{21}$ In line with our clinical data, plasma PCSK9 peaked at 2 days in an acute MI rat model ${ }^{22}$ in parallel with enhanced hepatic mRNA expression of PCSK9 and the transcription factors HNF1 $\alpha$ and SREBP-2, which may regulate the expression of $\mathrm{CRP}^{23}$ and promote inflammation in vascular cells ${ }^{24}$ respectively. However, we were unable to identify any correlation between PCSK9 and CRP or IL-6 levels, although a modest association with leucocyte numbers during hospitalisation was observed. Moreover, treatment with an anti-IL-6R antibody, which markedly attenuates the acute phase reactants (eg, decreases CRP, leucocyte counts), had no effect on PCSK9 levels in the study population as a whole. A lack of association between PCSK9 and CRP has been reported in two larger ACS populations, ${ }^{810}$ and stratification by use of lipid lowering therapy revealed significant associations in non-users in one study. ${ }^{8}$

In the largest study of PCSK9 in ACS, Gencer et al identified hypercholesterolaemia as a major determinant of PCSK9. ${ }^{8}$ In the present study, patients with medical history of hypercholesterolaemia had an enhanced response in PCSK9 during the acute phase, and notably, tocilizumab had a significant attenuating effect on serum PCSK9 in these but not in the other patients. In addition to its ability to inhibit IL-6-induced CRP production, blocking IL-6 signalling with tocilizumab is associated with transient leucopenia/neutropenia, ${ }^{25}$ as also found in the present NSTEMI study. ${ }^{17}$ However, while the leucopenia during hospitalisation was similar in patients treated with tocilizumab, irrespective of hypercholesterolaemia status, we observed a strong correlation between PCSK9

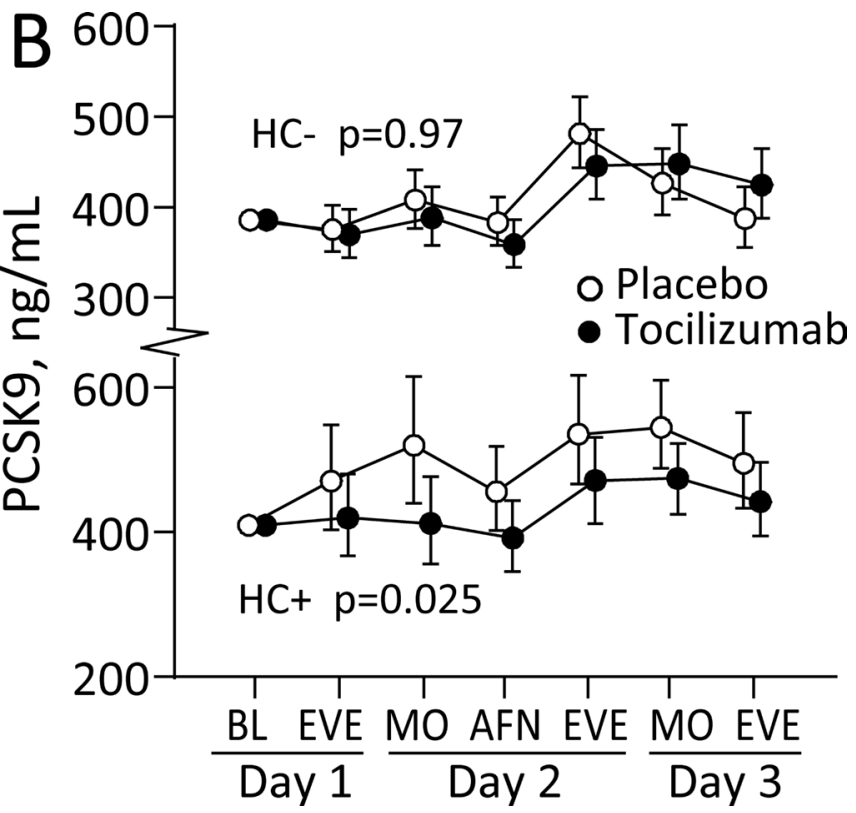

Figure 3 Serum PCSK9 during hospitalisation in non-ST-elevation myocardial infarction (NSTEMI) stratified by hypercholesterolaemia. (A) Comparison of AUC during hospitalisation for PCSK9 in groups stratified by treatment and presence of hypercholesterolaemia $(\mathrm{HC})$. Boxes represent median and 25th and 75th percentiles. (B) Serum PCSK9 during hospitalisation stratified by treatment and hypercholesterolaemia. The $p$ values in (B) represent the group effects from the repeated measures analysis of variance (ANOVA). Circles represent geometric back-transformed estimated marginal means $95 \%$ Cls. AFN, afternoon; AUC, area under the curve; BL, baseline; EVE, evening; MO, morning; PCSK9, proprotein convertase subtilisin-kexin type 9. 

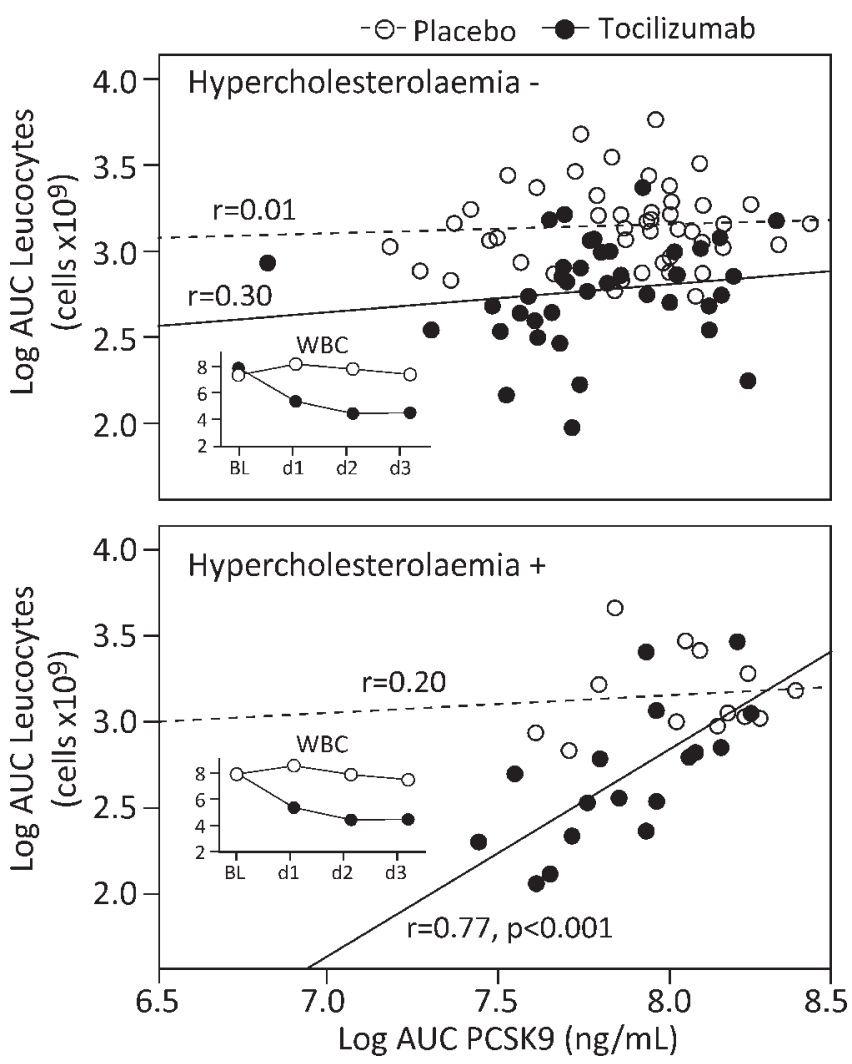

Figure 4 Correlation between AUC during hospitalisation for neutrophils and PCSK9 stratified by absence (top panel) or presence (bottom panel) of hypercholesterolaemia and treatment (clear circles, placebo; filled circles, tocilizumab). Correlation coefficients within each stratification group are given and $p$ value when significant. The small graph in each panel shows the average white cell count within each stratification group according to treatment during hospitalisation. AUC, area under the curve; BL, baseline; PCSK9, proprotein convertase subtilisin-kexin type 9.

and leucocyte count only in patients with hypercholesterolaemia in their medical history treated with tocilizumab. Experimental and clinical studies indicate that hypercholesterolaemia increases the number and atherogenic potential of neutrophils and monocytes ${ }^{26}$ and we speculate that the attenuating effect of tocilizumab on PCSK9 in hypercholesterolaemia could be attributed to a decrease in the number of activated leucocytes that may influence PCSK9.

This substudy has several limitations such as a rather low number of patients and a wide distribution in the time from symptom onset to study drug administration. Moreover, PCSK9 levels are influenced by statins ${ }^{27}$ which may disrupt its association with LDL-C and could explain the lack of association with lipids in our study. Thus, in our trial where the majority $(90 \%)$ of patients were treated with statins, probing associations in statinnaive patients was not possible although statin use was included in relevant analyses as a covariate. Finally, our study was not designed or powered to evaluate hypercholesterolaemia and the group sizes were modest for this demographic.
The present study did not identify any association between markers of inflammation and PCSK9 during the acute phase response in NSTEMI or an effect of anti-IL-6 therapy on PCSK9 levels. However, a modifying effect of tocilizumab on PCSK9 levels was observed in patients with hypercholesterolaemia in the medical history which may suggest that patients with a more atherogenic profile may benefit from anti-IL-6 therapy with regard to PCSK9. This hypothesis should be further tested in forthcoming larger studies targeting inflammation in patients with ACS, and if proven, it will further link inflammation and lipids as two interacting arms in the pathogenesis of CAD.

Author affiliations

${ }^{1}$ Research Institute of Internal Medicine, Oslo University Hospital Rikshospitalet, Oslo, Norway

${ }^{2}$ Institute of Clinical Medicine, University of Oslo, Oslo, Norway

${ }^{3} \mathrm{KG}$ Jebsen TREC, University of Troms $\emptyset$, Troms $\emptyset$, Norway

${ }^{4}$ Department of Clinic of Cardiology, St Olavs Hospital, Trondheim, Norway

${ }^{5}$ Department of Circulation and Medical Imaging, Norwegian University of Science and Technology (NTNU), Trondheim, Norway

${ }^{6}$ Centre of Molecular Inflammation Research, Department of Cancer Research and Molecular Medicine, Norwegian University of Science and Technology (NTNU), Trondheim, Norway

${ }^{7}$ Section of Clinical Immunology and Infectious Diseases, Oslo University Hospital Rikshospitalet, Oslo, Norway

${ }^{8}$ Department of Cardiology, Oslo University Hospital Rikshospitalet, 0slo, Norway ${ }^{9}$ Center for Heart Failure Research, University of Oslo, Oslo, Norway

Contributors TU and AEM performed the ELISA analyses. TU performed the statistical analysis and presentation of data. OK, RW, JKD, PA, LG and TU designed the study. TU, AY and BH initiated the current substudy. TU, AY and PA drafted the manuscript. All authors made critical revision and accepted the article in its current form.

Funding The authors have not given a specific grant for this research from any funding agency in the public, commercial or not-for-profit sectors.

Competing interests None declared.

Patient consent Obtained.

Ethics approval Regional Committee for Medical and Health Research Ethics of South-Eastern Norway and the Norwegian Medicines Agency.

Provenance and peer review Not commissioned; externally peer reviewed. Data sharing statement There are no additional data available for this paper.

Open access This is an open access article distributed in accordance with the Creative Commons Attribution Non Commercial (CC BY-NC 4.0) license, which permits others to distribute, remix, adapt, build upon this work non-commercially, and license their derivative works on different terms, provided the original work is properly cited, appropriate credit is given, any changes made indicated, and the use is non-commercial. See: http://creativecommons.org/licenses/by-nc/4.0/.

\section{REFERENCES}

1. Dadu RT, Ballantyne CM. Lipid lowering with PCSK9 inhibitors. Nat Rev Cardiol 2014:11:563-75.

2. Abifadel M, Rabès JP, Devillers M, et al. Mutations and polymorphisms in the proprotein convertase subtilisin kexin 9 (PCSK9) gene in cholesterol metabolism and disease. Hum Mutat 2009;30:520-9

3. Robinson JG, Farnier M, Krempf M, et al. Efficacy and safety of alirocumab in reducing lipids and cardiovascular events. $N$ Engl $J$ Med 2015;372:1489-99.

4. Sabatine MS, Giugliano RP, Keech AC, et al. Evolocumab and Clinical Outcomes in Patients with Cardiovascular Disease. N Engl $J$ Med 2017;376:1713-22.

5. Ferri N, Corsini A, Macchi C, et al. Proprotein convertase subtilisin kexin type 9 and high-density lipoprotein metabolism: experimental animal models and clinical evidence. Trans/ Res 2016;173:19-29.

6. Laugsand LE, Åsvold BO, Vatten LJ, et al. Circulating PCSK9 and Risk of Myocardial Infarction. JACC Basic Trans/ Sci 2016;1:568-75. 
7. Burchardt P, Rzeźniczak J, Dudziak J, et al. Evaluation of plasma PCSK9 concentrations, transcript of LDL receptor, as well as the total number of monocyte LDL receptors in acute coronary syndrome patients. Cardiol J 2016;23:604-9.

8. Gencer B, Montecucco F, Nanchen D, et al. Prognostic value of PCSK9 levels in patients with acute coronary syndromes. Eur Heart J 2016;37:546-53.

9. Navarese EP, Kolodziejczak M, Winter MP, et al. Association of PCSK9 with platelet reactivity in patients with acute coronary syndrome treated with prasugrel or ticagrelor: The PCSK9-REACT study. Int J Cardiol 2017;227:644-9.

10. Cariou B, Guérin P, Le May C, et al. Circulating PCSK9 levels in acute coronary syndrome: Results from the PC-SCA-9 prospective study. Diabetes Metab 2017;43:529-35.

11. Tang Z, Jiang L, Peng J, et al. PCSK9 siRNA suppresses the inflammatory response induced by oxLDL through inhibition of NF-kB activation in THP-1-derived macrophages. Int $\mathrm{J} \mathrm{Mol} \mathrm{Med}$ 2012;30:931-8.

12. Wu CY, Tang ZH, Jiang L, et al. PCSK9 siRNA inhibits HUVEC apoptosis induced by ox-LDL via Bcl/Bax-caspase9-caspase3 pathway. Mol Cell Biochem 2012;359(1-2):347-58.

13. Ferri N, Tibolla G, Pirillo A, et al. Proprotein convertase subtilisin kexin type 9 (PCSK9) secreted by cultured smooth muscle cells reduces macrophages LDLR levels. Atherosclerosis 2012;220:381-6.

14. Cheng JM, Oemrawsingh RM, Garcia-Garcia HM, et al. PCSK9 in relation to coronary plaque inflammation: Results of the ATHEROREMO-IVUS study. Atherosclerosis 2016;248:117-22.

15. Gabay C. Interleukin-6 and chronic inflammation. Arthritis Res Ther 2006;8 Suppl 2(Suppl 2):S3.

16. Ikeda U, Ito T, Shimada K. Interleukin-6 and acute coronary syndrome. Clin Cardiol 2001;24:701-4.

17. Kleveland O, Kunszt G, Bratlie M, et al. Effect of a single dose of the interleukin-6 receptor antagonist tocilizumab on inflammation and troponin T release in patients with non-ST-elevation myocardial infarction: a double-blind, randomized, placebo-controlled phase 2 trial. Eur Heart J 2016;37:2406-13.

18. Norheim OF, Gjelsvik B, Kjeldsen SE, et al, 2009. National guidelines for individual primary prevention of cardiovascular disease: Norwegian Directorate of Health https://helsedirektoratet.no/Lists/ Publikasjoner/Attachments/444/Nasjonal-retningslinje-for-individuellprim\%C3\%A6rforebygging-av-hjerte-og-karsykdommer-IS-1550.pdf

19. Almontashiri NA, Vilmundarson RO, Ghasemzadeh N, et al. Plasma PCSK9 levels are elevated with acute myocardial infarction in two independent retrospective angiographic studies. PLoS One 2014;9:e106294.

20. Feingold KR, Moser AH, Shigenaga JK, et al. Inflammation stimulates the expression of PCSK9. Biochem Biophys Res Commun 2008;374:341-4.

21. Le Bras M, Roquilly A, Deckert V, et al. Plasma PCSK9 is a late biomarker of severity in patients with severe trauma injury. $J$ Clin Endocrinol Metab 2013;98:E732-E736.

22. Zhang Y, Liu J, Li S, et al. Proprotein convertase subtilisin/kexin type 9 expression is transiently up-regulated in the acute period of myocardial infarction in rat. BMC Cardiovasc Disord 2014;14:192.

23. Armendariz AD, Krauss RM. Hepatic nuclear factor 1-alpha: inflammation, genetics, and atherosclerosis. Curr Opin Lipidol 2009;20:106-11.

24. Tall AR, Cholesterol Y-CL. inflammation and innate immunity. Nature reviews Immunology 2015;15:104-16.

25. Wright HL, Cross AL, Edwards SW, et al. Effects of IL-6 and IL-6 blockade on neutrophil function in vitro and in vivo. Rheumatology 2014:53:1321-31.

26. Soehnlein O, Drechsler M, Hristov M, et al. Functional alterations of myeloid cell subsets in hyperlipidaemia: relevance for atherosclerosis. J Cell Mol Med 2009;13(11-12):4293-303.

27. Welder G, Zineh I, Pacanowski MA, et al. High-dose atorvastatin causes a rapid sustained increase in human serum PCSK9 and disrupts its correlation with LDL cholesterol. J Lipid Res 2010;51:2714-21. 